\title{
Genetics of Anisotropy Asymmetry: Registration and Sample Size Effects
}

\author{
Neda Jahanshad ${ }^{1,2}$, Agatha D. Lee ${ }^{1}$, Natasha Leporé ${ }^{1}, Y_{i}-Y u$ Chou ${ }^{1}$, \\ Caroline C. Brun ${ }^{1}$, Marina Barysheva ${ }^{1}$, Arthur W. Toga ${ }^{1}$, Katie L. McMahon ${ }^{3}$, \\ Greig I. de Zubicaray ${ }^{3}$, Margaret J. Wright ${ }^{4}$, and Paul M. Thompson ${ }^{1}$ \\ 1 Laboratory of Neuro Imaging, Department of Neurology, UCLA, CA USA \\ 2 Medical Imaging Informatics Group, Department of Radiology, UCLA, CA USA \\ 3 University of Queensland, fMRI Laboratory, Centre for MR, Brisbane, Australia \\ ${ }^{4}$ Queensland Institute of Medical Research, Brisbane, Australia
}

\begin{abstract}
Brain asymmetry has been a topic of interest for neuroscientists for many years. The advent of diffusion tensor imaging (DTI) allows researchers to extend the study of asymmetry to a microscopic scale by examining fiber integrity differences across hemispheres rather than the macroscopic differences in shape or structure volumes. Even so, the power to detect these microarchitectural differences depends on the sample size and how the brain images are registered and how many subjects are studied. We fluidly registered 4 Tesla DTI scans from 180 healthy adult twins (45 identical and fraternal pairs) to a geometrically-centered population mean template. We computed voxelwise maps of significant asymmetries (left/right hemisphere differences) for common fiber anisotropy indices (FA, GA). Quantitative genetic models revealed that $47-62 \%$ of the variance in asymmetry was due to genetic differences in the population. We studied how these heritability estimates varied with the type of registration target (T1- or T2-weighted) and with sample size. All methods consistently found that genetic factors strongly determined the lateralization of fiber anisotropy, facilitating the quest for specific genes that might influence brain asymmetry and fiber integrity.
\end{abstract}

\section{Introduction}

Asymmetries in brain structure and function have been the topic of neuroimaging studies for many years. Anatomical asymmetries may help to reveal the origins of lateralized cognitive functions or behavioral traits, such as language and handedness, that may arise from partially genetic hemispheric differences during development [1. Studies of brain asymmetry can also inform clinical research, as aberrant asymmetries have been hypothesized or detected in disorders such as schizophrenia, dyslexia, or hemiparesis, which may arise from a derailment in processes that establish normal brain lateralization and hemispheric specialization. Deformation-based morphometry studies have used the theory of random Gaussian vector fields to detect statistical departures from the normal level of brain asymmetry [2]. 
Many imaging studies have used MRI to study brain asymmetries, but very few have used DTI. In DTI, the MR signal attenuation due to water diffusion in direction $k$ decreases according to the Stejskal-Tanner equation: $S_{k}(\mathbf{r})=$ $S_{0}(\mathbf{r}) e^{-b_{k} D_{k}(\mathbf{r})}$ where $S_{0}(\mathbf{r})$ is the non-diffusion weighted baseline intensity, $D_{k}(\mathbf{r})$ is the apparent diffusion coefficient (ADC), and $b_{k}$ is Le Bihan's factor; the fractional and geodesic anisotropy (FA and GA), calculated from a local tensor approximation for $D_{k}(\mathbf{r})$, are commonly used measures of fiber integrity; FA correlates highly with IQ (intelligence quotient) in normal subjects [3].

Previous DTI asymmetry studies have focused on specific tracts (e.g., the corticospinal tract [4, and the arcuate fasciculus involved in language processing [56]). Frontal and temporal white matter show left greater than right FA even in early infancy 7, suggesting greater myelination in the left hemisphere 77. Frontal FA differences between the two hemispheres diminish as the brain develops, but temporal lobe asymmetries persist 8 .

Studies of asymmetries in white matter characteristics may be confounded by the vast structural asymmetries present. In frontal and occipital regions, the natural petalia (torquing) of the brain shifts the right hemisphere structures anterior to their left hemisphere counterparts 1. Men may have greater anatomical asymmetries than women [1, making it advantageous to reduce these pronounced macrostructural differences when gauging the level of microstructural asymmetry in a mixed-sex population.

Twin studies have long been used to determine genetically and environmentally influenced human traits. Monozygotic twins share all their genes while dizygotic twins share, on average, half. Estimates of the proportion of variance attributable to genes versus environment can be inferred by fitting structural equation models to data from both types of twins. Twin neuroimaging studies reveal that genetic factors strongly influence several aspects of brain structure, e.g., cortical thickness, and gray and white matter volumes [9], but twin studies using DTI are rare.

Here we created the first DTI-based maps of asymmetries (left/right hemisphere differences) in fiber characteristics (FA, GA) in a large twin population $(\mathrm{N}=180)$. We adjusted, as far as possible, for the known structural differences between hemispheres by aligning brains to a symmetrized minimal deformation target (MDT) created from all of the images.

The choice of registration target is known to affect the accuracy of region of interest (ROI) analyses [10, so we evaluated the effects of using different registration targets based on the separate structural MRI images, including (1) an MDT created by geometrically adjusting an individual subject's image, (2) a population-averaged MDT, and (3) a population-averaged MDT based on the non-diffusion-sensitized T2-weighted images collected as part of the DTI protocol. We then determined whether genetic factors influenced the residual asymmetries, and examined the stability of the estimates with respect to sample size and the choice of registration target. 


\section{Methods}

\subsection{Image Acquisition and Subject Information}

Structural and diffusion tensor (DT) MRI scans were acquired from 180 subjects using a high magnetic field (4T) Bruker Medspec MRI scanner. T1-weighted images were collected using an inversion recovery rapid gradient echo sequence, with parameters: TI/TR/TE $=1500 / 2500 / 3.83$ msec; flip angle=15 degrees; slice thickness $=0.9 \mathrm{~mm}$, and $256 \times 256 \times 256$ acquisition matrix. Diffusion-weighted images were also acquired using 30 gradients (27 diffusion-weighted images and 3 with no diffusion sensitization; i.e., T2- weighted images) with gradient directions uniformly distributed on the hemisphere. Parameters were: $23 \mathrm{~cm}$ FOV, TR/TE 6090/91.7ms, b-value $=1132 \mathrm{~s} / \mathrm{mm}^{2}$, scan time: 3.05 minutes. Each 3D volume consisted of 215 -mm thick axial slices with a $0.5 \mathrm{~mm}$ gap and $1.8 \times 1.8 \mathrm{~mm}^{2}$ in-plane resolution. The subjects included 90 young adult monozygotic (MZ) twins and 90 dizygotic (DZ) same sex twins (45 pairs of each). All subjects were right-handed young adults (average age 24.37, stdev 1.936).

\section{$2.2 \quad$ Creating Templates}

To determine whether asymmetric differences are influenced by the template used for registration, several templates were created and compared. Three templates were created using the T1-weighted images to help adjust for the structural differences across subjects and hemispheres, and another template was created from the T2-weighted images acquired along with the diffusion weighted scans, which are in perfect register with the diffusion tensor data. T1-weighted structural MR images were edited to remove extracerebral tissues and were linearly registered to a symmetrical template. This symmetrical template was created by averaging a high-resolution single subject average scan, the Colin27 [11, with the same image reflected in the midsagittal plane. This centered each subjects midline within the image volume. All subjects images were linearly registered to the symmetrical template using FLIRT software http://fsl.fmrib.ox.ac.uk/ fsl/flirt with 9-parameter registration and a correlation ratio cost function.

T1 Template 1(non-symmetric). One minimal deformation target (MDT) was created using only the original scan orientations, using non-linear fluid registration as described in [12]13. This template was not symmetrical as all the images used to create it were of the original orientation. MDTs were created using the method proposed by Kochunov [14] (although alternative methods are possible): the $N$ 3D vector fields fluidly registering a specific individual to all other subjects were averaged and applied to that subject, geometrically adjusting their anatomy, but retaining the image intensities and anatomical features of that specific subject. T1 Template 2 (initial symmetrization). Linearly aligned subject images were reflected over the midline to produce a mirrored set. Another MDT was then created from four independent (one per pair) monozygotic (MZ) twins and four independent dizygotic (DZ) twin image volumes randomly selected with their corresponding reflected images. These 16 image sets were 
then used to generate an MDT using fluid registration as described in [12 13]. The flipped images of the same brains were included during MDT construction to make it symmetric.

T1 Template 3 (symmetric population averaged MDT). A populationaveraged MDT was created to further reduce the structural asymmetries. 8 separate MDTs were constructed as described above, each formed from 6 subjects and their corresponding images flipped over the midline. For 4 of these MDTs, the initial template image was in the original orientation while for the other 4 , the template was in the flipped orientation. All 8 MDTs were then averaged together to produce the population averaged MDT, incorporating T1 information from 50 independent subjects.

T2 Template (symmetric population averaged MDT). Another population averaged MDT was constructed from the T2-weighted images, in the same manner as for the T1-weighted population MDT, with the same set of subjects. All subjects' images were first linearly aligned to a single subject image. This image of the single subject was aligned such that the midsagittal plane of the brain was centered. Another image was created by mirroring the result in the midsagittal plane. This flipped image was averaged with its original to create a symmetric template to linearly align all the T2-weighted scans and their mirror images before creating the MDT.

Structural T1 images from 100 subjects (25 MZ, 25 DZ pairs) were then fluidly registered to each of the 3 T1-weighted MDTs using a 3D Navier-Stokes-based fluid warping technique enforcing diffeomorphic mappings, using least squares intensity differences as a cost function [1213. T2-weighted images for each of the 180 subjects were registered to the T2-weighted MDT with the same technique. $3 \mathrm{D}$ deformation fields for all mappings were retained.

\subsection{Anisotropy Asymmetry Maps}

Diffusion tensors were computed from the diffusion-weighted images using MedINRIA software http://www.sop.inria.fr/asclepios/software/MedINRIA.

Scalar images of anisotropy measures were created for each of the 180 subjects from the eigenvalues $\left(\lambda_{1}, \lambda_{2}, \lambda_{3}\right)$ of the symmetric $3 x 3$ diffusion tensor. These included the fractional anisotropy (FA), geodesic anisotropy (GA) computed in the Log-Euclidean framework [15], hyperbolic tangent of the GA (tGA), to take values in the same range as $\mathrm{FA}$, i.e., $[0,1]$, and mean diffusivity (MD):

$$
\begin{gathered}
F A=\sqrt{\frac{3}{2}} \frac{\sqrt{\left(\lambda_{1}-\hat{\lambda}\right)^{2}+\left(\lambda_{2}-\hat{\lambda}\right)^{2}+\left(\lambda_{3}-\hat{\lambda}\right)^{2}}}{\sqrt{\lambda_{1}^{2}+\lambda_{2}^{2}+\lambda_{3}^{2}}}, \hat{\lambda}=M D=\frac{\lambda_{1}+\lambda_{2}+\lambda_{3}}{3} \\
G A(S)=\sqrt{\operatorname{Trace}(\log S-<\log S>I)^{2}}, \quad\left\langle\log S>=\frac{\operatorname{Trace}(\log S)}{3}\right.
\end{gathered}
$$

Extra-cerebral tissue was manually deleted from one directional component of the diffusion tensors $\left(D_{x x}\right)$ creating a mask that was then applied to the scalar anisotropy maps created for each subject. Once masked, these anisotropy images 
were then linearly aligned to the symmeterized templates and fluidly registered to each of the MDTs by applying the deformation fields described in Section 2.2.

Each aligned anisotropy map was then mirrored across midline, and the voxelwise difference map between the original and flipped images was created. In this new map, the left side of the image represents the difference between the subjects right and left hemispheres; voxels on the other side of the image have the opposite sign. Maps were obtained of the percent difference between the resulting difference image and the average of the two mirror image orientations.

\subsection{Calculating Genetic Contributions}

Voxel-wise maps of the intra-class correlations (ICC) within MZ and DZ twins, $r_{M Z}$ and $r_{D Z}$ respectively, were derived as well as Falconer's heritability estimate, $h^{2}=2\left(r_{M Z}-r_{D Z}\right)$ [16] for the asymmetry in FA, GA, tGA and MD.

Average measures of the anisotropy difference were examined in certain regions of interest (ROIs). We determined the genetic contribution to the asymmetries in each lobe of the brain. ROIs were traced for the four lobes (frontal, parietal, temporal, and occipital) in one hemisphere of each MDT and were flipped to define the same ROI in the opposite hemisphere. This ensured consistency between hemispheres and reduced errors due to manual labeling. For each anisotropy measure, covariances for the average ROI values in pairs of MZ and DZ twins were entered into a univariate structural equation model to estimate additive genetic (A), shared environmental (C) and unique environmental (E) components of the variance in asymmetry [17. Mx modeling software http://www.vcu.edu/mx/ was used.

This form of structural equation modeling finds the maximum likelihood estimate (eq. 3) for $\Sigma$ ( $\alpha=1$ for MZ and 0.5 for DZ) to estimate genetic versus environmental contributions to the variance, where $S_{g}$ is the observed covariance matrix for each twin group $g$ :

$$
M L_{g}=N_{g}\left\{\ln \left|\Sigma_{g}\right|-\ln \left|S_{g}\right|+\operatorname{tr}\left(S_{g} \Sigma_{g}^{-1}\right)-2 m\right\}, \Sigma=\left[\begin{array}{cc}
a^{2}+c^{2}+e^{2} & \alpha a^{2}+c^{2} \\
\alpha a^{2}+c^{2} & a^{2}+c^{2}+e^{2}
\end{array}\right]
$$

\section{Results}

Figure 1A shows the mean FA asymmetry as a percent difference between left and right hemispheres, relative to which genetic effects were determined. Frontal and temporal regions show high asymmetry $(\sim 25 \%, p<0.05)$. Frontal FA is higher in the right hemisphere, while temporal FA is higher on the left. The asymmetries found in the temporal lobe correspond to language centers 1] consistent with [78. The magnitude of the asymmetry difference is somewhat dependent on the number of subjects used in the study, but patterns are largely consistent. Figure 1B shows differences arising in ICC and Falconer's heritability estimates when

using the T1-weighted population template for 100 subjects and the T2-weighted MDT for the different population sizes. Despite evidence for some subcortical effects, voxelwise maps are somewhat noisy even with $N=180$ subjects, partly 

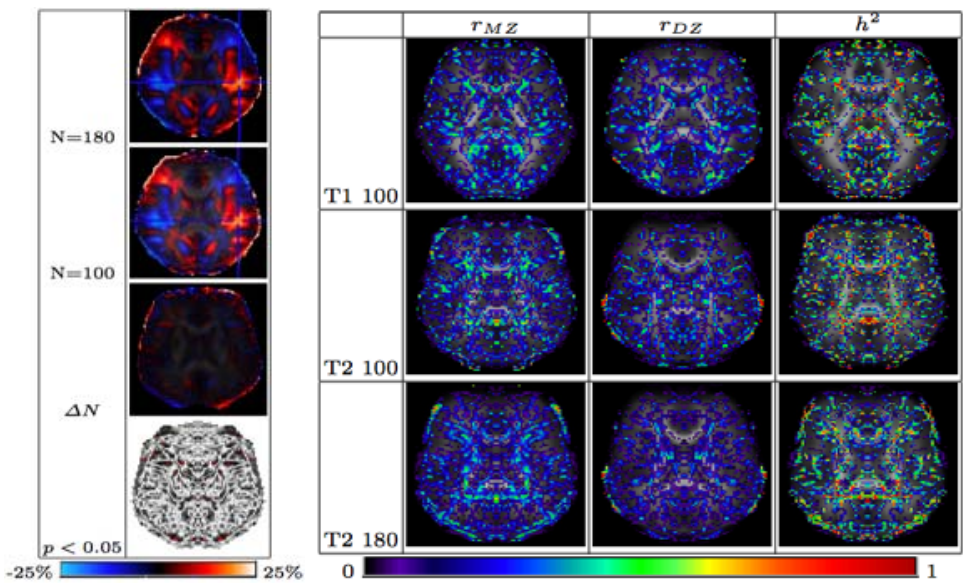

Fig. 1. A: The mean asymmetry in FA, in a sample of $\mathrm{N}=180$ subjects, reaches $25 \%$ in frontal and temporal regions. The localization of results based on 180 vs only 100 subjects is largely consistent, as shown by the difference image and the image of the $p$-values. B: ICC and Falconer's $h^{2}$ maps for asymmetries in FA images. Top: FA results of 100 subjects mapped to the population-averaged T1 MDT; Center: results from 100 subjects mapped to population-averaged T2 MDT; Bottom: results from 180 subjects mapped to the T2 MDT
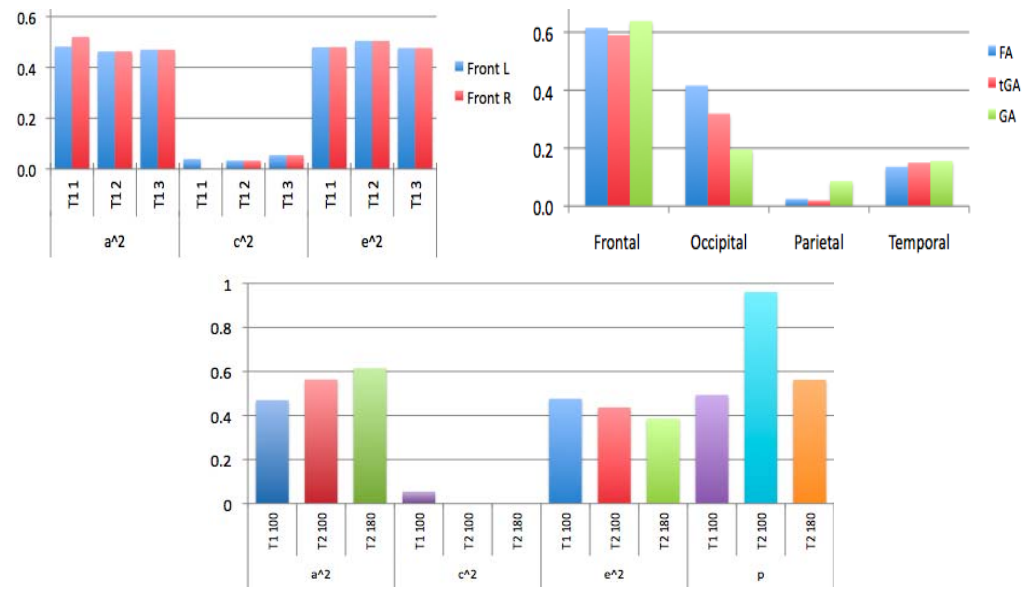

Fig. 2. A/C/E Genetic effects: Top Left: Symmetrization Effects:ACE results showing genetic and environmental contributions of template choice asymmetry in FA; Top Right: Frontal Lobe FA ACE results of using the population averaged T1 template (100 subjects) and T2 template (100 and 180 subjects) for FA asymmetry in the frontal lobe. $p$-values derived from $\chi^{2}$ statistics show the ACE model fits well in all cases $(p>0.05)$; Bottom: $N=180$ Genetic Effects genetic component of variance (A) determined from mapping 180 subjects to the T2-weighted MDT for all anisotropy measures, in each lobe. Genetic effects are greatest in lobes with the highest mean asymmetries (Fig. 1). 

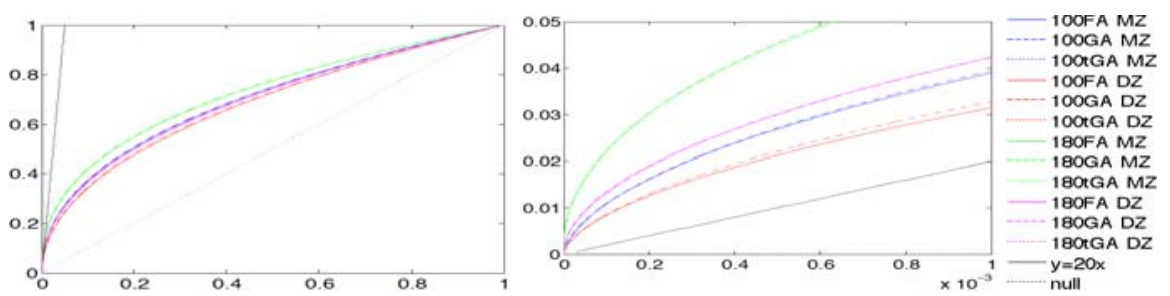

Fig. 3. CDF of significant $p$-values for anisotropy asymmetries mapped to the T2weighted population MDT for 100 (left) and 180 subjects (right)

because $h^{2}$ is a difference in correlations. We therefore summarize FA asymmetry in lobar ROIs, to increase power for genetic analyses.

Figure 2 shows genetic $\left(a^{2}\right)$ vs environmental $\left(c^{2}, e^{2}\right)$ effects on FA asymmetry. Intriguingly, the asymmetry in frontal lobe mean FA was $\sim 50 \%$ determined by genetic factors, with no evidence for a shared environmental effect $\left(c^{2} \sim 0 \%\right)$. The $e^{2}$-term contains registration errors as well as unique effects, so there is some evidence that using 180 (vs 100) subjects, and using a T2 vs T1 template, more accurately captures the true genetic contributions to these asymmetries, as the $e^{2}$-term is slightly lower. In structural equation models, $p>0.05$ denotes that the ACE model fits well. All models here yield a good fit.

Figure 3 plots the cumulative distribution function (cdf) of the p-values associated with the ICC against those that would be expected from a null distribution. As the cdf initially rises faster than 20 times the null, we are able to reasonably claim significance at the $5 \%$ level. For null distributions (i.e. no group difference detected), these are expected to fall along the $\mathrm{x}=\mathrm{y}$ line, and larger deviations from that curve represent larger effect sizes.

\section{Discussion}

In this study, we examined the genetic and environmental contributions to the differences in fiber integrity across brain hemispheres. Genetic factors determined about half of the variance in these asymmetries, with greatest effects in the frontal and occipital lobes, where mean asymmetries were greatest (reaching $25 \%$ ) (Fig. 1A). Interestingly, strong genetic effects (significant ACE models) were detectable for anisotropy indices (FA, GA). Results were stable when the images were fluidly registered to various different anatomical templates, including ones constructed to have hemispheric symmmetry. These results suggest that specific genetic factors determining hemispheric asymmetries in fiber architecture may be identifiable in very large samples.

Acknowledgments. Supported by grants from the NLM, NIH and NICHD. 


\section{References}

1. Toga, A., Thompson, P.: Mapping brain asymmetry. Nat. Rev. Neurosci. 4(1) (2003)

2. Thirion, J.P., Prima, S., Subsol, G., Roberts, N.: Statistical analysis of normal and abnormal dissymmetry in volumetric medical images. Med. Im. Analy. 4(2) (2000)

3. Chiang, M., Barysheva, M., Lee, A., Madsen, S., Klunder, A., Toga, A., McMahon, K., de Zubicaray, G., Wright, M., Srivastava, A., Balov, N., Thompson, P.: Genetics of brain fiber architecture and intelligence. Journal of Neuroscience (2009)

4. Westerhausen, R., Huster, R.J., Kreuder, F., Wittling, W., Schweiger, E.: Corticospinal tract asymmetries at the level of the internal capsule: Is there an association with handedness? Neuroimage 37(2), 379-386 (2007)

5. de Jong, L., Kovacs, S., Bamps, S., Calenbergh, F.V., Sunaert, S., van Loon, J.: The arcuate fasciculus: a comparison between diffusion tensor tractography and anatomy using the fiber dissection technique. Surgical Neurology 71(1) (2009)

6. Rodrigo, S., Naggara, O., Oppenheim, C., Golestani, N., Poupon, C., Cointepas, Y., Mangin, J.F., Le Bihan, D., Meder, J.F.: Human subinsular asymmetry studied by diffusion tensor imaging and fiber tracking. AJNR 28(8), 1526-1531 (2007)

7. Dubois, J., Hertz-Pannier, L., Cachia, A., Le Bihan, D., Dehaene-Lambertz, G.: Structural asymmetries in the infant language and sensori-motor networks. Cerebral Cortex 19(2), 414-423 (2008)

8. Barnea-Goraly, N., Menon, V., Eckert, M., Tamm, L., Bammer, R., Karchemskiy, A., Dant, C.C., Reiss, A.L.: White matter development during childhood and adolescence: A cross-sectional diffusion tensor imaging study. Cereb. Cortex 15(12), 1848-1854 (2005)

9. Pfefferbaum, A., Sulluvan, E.V., Carmelli, D.: Genetic regulation of regional microstructure of the corpus callosum in late life. Neuroreport 12(8), 1677-1681 (2001)

10. Wang, Q., Seghers, D., D’Agostino, E., Maes, F., Vandermeulen, D., Suetens, P., Hammers, A.: Construction and validation of mean shape atlas templates for atlasbased brain image segmentation. In: Christensen, G.E., Sonka, M. (eds.) IPMI 2005. LNCS, vol. 3565, pp. 689-700. Springer, Heidelberg (2005)

11. Holmes, C.J., Hoge, R., Collins, L., Woods, R., Toga, A.W., Evans, A.C.: Enhancement of MR images using registration for signal averaging. J. Comput. Assist. Tomogr. 22(2), 324-333 (1998)

12. Leporé, N., Brun, C., Pennec, X., Chou, Y.Y., Lopez, O., Aizenstein, H., Becker, J., Toga, A., Thompson, P.: Mean template for tensor-based morphometry using deformation tensors. In: Ayache, N., Ourselin, S., Maeder, A. (eds.) MICCAI 2007, Part II. LNCS, vol. 4792, pp. 826-833. Springer, Heidelberg (2007)

13. Leporé, N., Chou, Y.Y., Lopez, O.L., Aizenstein, H.J., Becker, J.T., Toga, A.W., Thompson, P.M.: Fast 3D fluid registration of brain magnetic resonance images, vol. 6916. SPIE, San Diego (2008)

14. Kochunov, P., Lancaster, J., Thompson, P., Toga, A., Brewer, P., Hardies, J., Fox, P.: An optimized individual target brain in the Talairach coordinate system. Neuroimage 17(2), 922-927 (2002)

15. Arsigny, V., Fillard, P., Pennec, X., Ayache, N.: Log-Euclidean metrics for fast and simple calculus on diffusion tensors. MRM 56(2), 411-421 (2006)

16. Falconer, D., Macka, T.F.: Introduction to Quantitative Genetics, 4th edn. Addison Wesley Longman, Amsterdam (1995) (Pearson Education)

17. Rijsdijk, F.V., Sham, P.C.: Analytic approaches to twin data using structural equation models. Briefings in Bioinformatics 3(2), 119-133 (2002) 